\title{
On the Design of LAS Spreading Codes
}

\author{
Byoung-Jo Choi and Lajos Hanzo ${ }^{1}$ \\ Dept. of ECS, University of Southampton, SO17 1BJ, UK. \\ Tel: +44-23-8059-3125, Fax: +44-23-8059-4508 \\ Email:1h1@ecs.soton.ac.uk, http://www-mobile.ecs.soton.ac.uk
}

\begin{abstract}
A family of Large Area Synchronised (LAS) codes is studied, which exhibits a so-called Interference Free Window (IFW), where both the Inter Symbol Interference (ISI) and the Multiple User Interference (MAI) are suppressed, provided that the relative time offset of the codes is within the IFW. Hence, LAS codes have the potential of increasing the capacity of CDMA networks. However, a specific drawback of this family of sequences is their relatively low duty ratio. A modified algorithm is proposed for determining the constituent LA code's pulse positions for the sake of improving the duty ratio of the corresponding LAS codes. It is shown that the modified LAS codes exhibit an $18.6 \%$ higher duty ratio, than that of the original LAS codes, when considering a minimum LA code pulse interval of 38. The LAS code design examples provided revealed that the system employing the proposed approach improved the data throughput by $\mathbf{4 0 \%}$ for the scenario, when the required IFW is \pm 4 -chips and the minimum pulse interval is 19. The availability of the LAS codes is also investigated in conjunction with for the various $L S$ and LA code lengths, employing an extended LS code construction scheme for polyphase complementary sequences and orthogonal LA pulse codes. It is shown that these modified spreading code construction schemes can be combined for generating a family of LAS codes in order to meet various design requirements.
\end{abstract}

\section{INTRODUCTION}

The spreading sequences employed characterise the Inter Symbol Interference (ISI) as well as the Multiple Access Interference (MAI) properties of Direct Sequence (DS) Code Division Multiple Access (CDMA) systems [1]. The traditional spreading sequences [2], such as $m$-sequences, Gold codes and Kasami codes, exhibit non-zero off-peak autocorrelations and crosscorrelations, which limits the achievable performance in asynchronous scenarios. Hence, a considerable research efforts have been invested in designing spreading sequences, which exhibit zero correlation values, when the relative code offset is in the so-called Zero Correlation Zone (ZCZ) or Interference Free Window (IFW) [3]. If we allow a guard interval between the transmission of spread symbols such that it is longer than the maximum channel-induced delay spread, all the correlation properties of spreadin codes are characterised by their aperiodic correlations rather than by their periodic correlations.

The attractive family of Large Area Synchronised (LAS)CDMA spreading sequences is constituted by the combination

The financial support of LGE, Korea; The CEC, Brussels; EPSRC, UK; and that of the Mobile VCE, UK is gratefully ackowledged. of the so-called Large Area (LA) codes $[4,5]$ and Loosely Synchronous (LS) codes [6]. LAS codes exhibit an IFW, where the off-peak aperiodic autocorrelation values as well as the aperiodic crosscorrelation values become zero, resulting in zero ISI and zero MAI, provided that the code-offset is within the IFW. In order to ensure that the relative offsets between the codes are within the IFW, the mobiles are expected to operate in a pseudo-synchronous manner. More specifically, interferencefree CDMA communications become possible, when the total time offset expressed in terms of the number of chip intervals, whcih is the sum of the time-offset of the mobiles plus the maximum channel-induced delay spread is within the designed IFW. Li [4] studied various LA code construction schemes and their application to CDMA system. Stańczak, Boche and Haardt [6] also investigated the construction of various schemes designed for the generation of various LS codes. They also developed a systematic way of constructing so-called LS-like codes, which exhibit small aperiodic correlations within a specified relative offset window [7].

The aim of this contribution is to improve the properties of known design schemes applicable to LA [5], LS [6] and LAS codes in an effort to generate efficient, flexible LAS codes. Firstly, we focus our attention on the design of the LA codes in the next section. Then, the family of binary LS codes is extended to the set of polyphase LS codes in Section 3. Some LAS code design examples are compared and the improved properties are investigated in Section 4. Finally, Section 5 concludes with a summary of our results.

\section{LA CODES}

LA codes $[4,5]$ belong to a family of ternary codes having the elements of \pm 1 or 0 . Their maximum correlation magnitude is unity and they also exhibit an IFW. Let us denote the family of the $K$ number of orthogonal ternary codes employing $K$ number of binary \pm 1 pulses by $\operatorname{LA}(L, M, K)$, which exhibit a minimum spacing of $M$-chip duration between nonzero pulses, while having a code length of $L$. All the codes in an LA code family share the same legitimate pulse positions. As an example, the construction of the $\operatorname{LA}(847,38,16)$ code was described in $[4,5]$, where the 16 pulse positions, $p_{k}$, $k=0, \cdots, 15$, are given by:

$$
\begin{aligned}
\left\{p_{k}\right\}= & \{0,38,78,120,164,210,258,308, \\
& 360,414,470,530,592,660,732,808\} .
\end{aligned}
$$

A. Legitimate pulse positions and the required code length The legitimate pulse positions of LA codes are selected such that the maximum off-peak autocorrelation magnitude becomes unity. Li's original scheme [4] proposed for determining the 
LA pulse positions will be introduced first and then a modified algorithm will be presented, which is capable of increasing the duty ratio of the LA codes.

Let us define the pulse spacing $d_{k}$, which is related to the difference of the pulse positions, as:

$$
d_{k} \triangleq \begin{cases}p_{k+1}-p_{k} & \text { for } 0 \leq k<K-1 \\ L-p_{K-1} & \text { for } k=K-1\end{cases}
$$

The constraints imposed on the pulse spacing $d_{k}$ of the LA code are described in [4] as follows:

(a) $d_{k}$ should be even except for $d_{K-1}$,

(b) $d_{k} \neq d_{k^{\prime}}$ for $0 \leq k \neq k^{\prime}<K$ and

(c) $\sum_{k \in \mathbf{S}} d_{k} \neq \sum_{k^{\prime} \in \mathbf{S}^{\prime}} d_{k}^{\prime}$ for any $\mathbf{S}, \mathbf{S}^{\prime} \subset\{k \mid 0 \leq k<K\}$. These three constraints form a sufficient condition, guaranteeing that the number of pulses satisfying $p_{k}+n=p_{k^{\prime}}$ is at most one for $0<n<L$ and $0 \leq k, k^{\prime}<K$. It can be shown that hence the conditions (a) - (c) gurantee that the maximum correlation magnitude becomes unity.

However, careful investigation of the conditions satisfying the maximum correlation constraint of unity reveals that $d_{k}$ may not have to be always even. Based on this observation we employed the following simple algorithm for finding the legitimate pulse positions for the set of $\operatorname{LA}(L, M, K)$ codes:

$p_{0}=0, p_{1}=M$;

$$
\begin{aligned}
& \text { for }(k=2 ; k<K ; k++) \quad\{ \\
& \quad p_{k}=p_{k-1}+1 ; \\
& \quad \text { until }\left(\max _{n}\left\{R_{w_{k}}[n]\right\} \leq 1\right) p_{k}++;
\end{aligned}
$$

where the subscript $w_{k}$ of $R_{w_{k}}$ is a candidate code to be tested, whether it satisfies our desired property of having a unity autocorrelation, i.e. the $\operatorname{LA}\left(p_{k}+M+1, M, k+1\right)$ code having $k+1$ number of +1 pulses at the pulse positions $\left\{p_{k}\right\}$ and $R_{w_{k}}[n]$ is the aperiodic autocorrelation of $w_{k}$ at the offset of $n$ chip durations. Since this algorithm does not require $p_{k}$ to be even, the LA code length $L$ can be reduced in comparison to that generated by original LA code construction scheme of [4]. Figure 1 depicts the length $L$ of the LA codes constructed

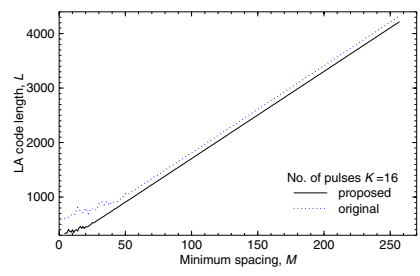

(a) Number of pulses $K=16$

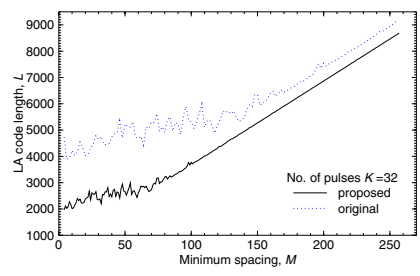

(b) Number of pulses $K=32$
Figure 1: The length $L$ of the family of $\operatorname{LA}(L, M, K)$ codes employing the proposed pulse positions and Li's original pulse positions [4]

employing the proposed legitimate pulse position search algorithm for various minimum pulse spacing values $M$ and for 16 as well as 32 pulses. When the minimum pulse spacing was $M=38$ and the number of pulses was $N=16$, the proposed algorithm required an LA code of length of $L=714$ chip durations in comparison to the original code length of $N=847$ chips proposed in $[4,5]$, resulting in an $18.6 \%$ duty ratio increase, as seen in Figure 1(a). By contrast, when the number of

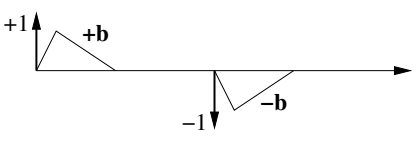

(a) Absolute encoding

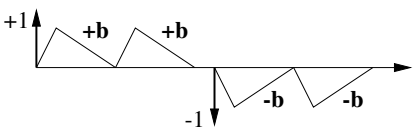

(c) Relative encoding - B

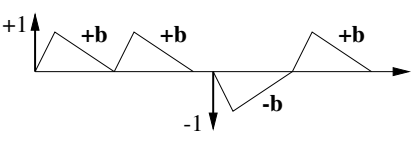

(b) Relative encoding - A

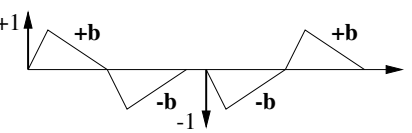

(d) Relative encoding - C
Figure 2: Various combining schemes for LA codes with the aid of a short sequence $\mathbf{b}$

pulses was 32, the new algorithm required an LA code length of 2478 chips, which corresponds to an $81 \%$ duty ratio increase in comparison to Li's original design of [5].

\section{B. Orthogonal codes and the number of pulses $K$}

The family of $K$ number of $\operatorname{LA}(L, M, K)$ codes employs a set of $K$ orthogonal binary pulses at $K$ number of legitimate predetermined pulse positions, derived from a $K \times K$ orthogonal binary matrix. The orthogonal code lengths $K$ in the LA code design examples of $[4,5]$ was $K=16$ and 32 . However, the length $K$ does not have to be $2^{n}$, where $n$ is a positive integer. It is conjectured that there exist binary orthogonal codes of length $K=4 n$ and this has been shown for code length values of up to $K<428$ [8]. In fact, Paley construction scheme of [9] can be employed for designing orthogonal codes for most of the scenarios, where $K=4 n$ is satified. When $K=2^{n}$, Sylvester construction scheme [9] generates the well-known family of Walsh-Hadamard codes.

\section{Extension schemes}

As a further code design improvement, the family of $\mathrm{LA}(L, M, K)$ codes can be combined with a secondary short code in an effort to increase its duty ratio. Two such spreadin code combining schemes were proposed in [5], which are illustrated in Figure 2(a) and 2(b). Specifically, Figure 2(a) illustrates the so-called absolute encoding scheme of [5], where additionally a short spreading sequence $\mathbf{b}$ is copied into a pulse position of an LA code where a positive pulse is hosted, while its negated version, namely $-\mathbf{b}$, is implanted into a negative pulse position. In the 'relative encoding scheme- $A$ ' of [5] shown in Figure 2(b) a set of two concatenated sequences composed of the same short sequence, namely $\mathbf{b b}$, is copied into a positive pulse position. By constrast, a concatenated sequence composed of a negated version followed by the original short sequence, namely $-\mathbf{b b}$, is implanted in a negative pulse position. Naturally, we can also derive the other two possible variations of the relative encoding scheme-A, which are illustrated in Figure 2(c) and 2(d).

For example, when we use the 13-bit Barker sequence [10] of $\mathbf{b}=\{+1+1+1+1+1-1-1+1+1-1+1-1+1\}$ as the additional short sequence $\mathbf{b}$ and apply the absolute encoding scheme of Figure 2(a), the duty ratio of the resultant combined codes increases by a factor 13 , yielding $16 \times 13 / 847=0.246$. One can further double the duty ratio to 0.49 by employing one of the relative encoding schemes of Figure 2 in comparison to 
the absolute encoding scheme of Figure 2(a). Figure 3 depicts the autocorrelations of the combined $\operatorname{LA}(847,38,16)$ codes in conjuction with the 13-bit Barker sequence employing the four different encoding schemes of Figure 2.

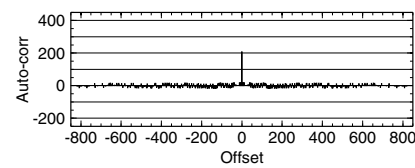

(a) Absolute encoding

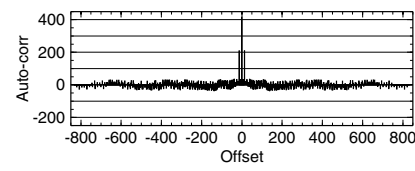

(c) Relative encoding - B

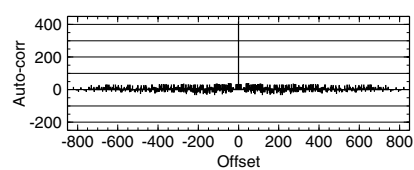

(b) Relative encoding - A

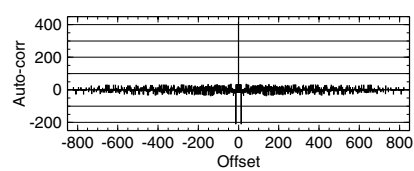

(d) Relative encoding - C
Figure 3: Autocorrelation of $R_{\mathrm{w}_{1}}$ for the combined $\mathrm{LA}(847,38,16)$ codes using the 13-bit Barker sequence employing the four different encoding schemes of Figure 2

The peak of the autocorrelation at zero offset was 208 in Figure 3(a), since the number of pulses of the combined codes becomes $16 \times 13=208$, when using the absolute encoding scheme of the 13-bit Barker sequence combined with the 16pulse LA code. The maximum off-peak autocorrelation magnitude in Figure 3(a) was in the range of 17 to 19 except for $R_{\mathrm{w}_{0}}$, which exhibited the maximum off-peak magnitude of 24 . On the other hand, when the relative encoding-A scheme of Figure 2(b) was used, the peak autocorrelation value at zero offset became $16 \times 13 \times 2=416$ and the maximum off-peak autocorrelation value was in the range of 34 to 38 except for $R_{\mathrm{w}_{0}}$, hence preserving the peak-to-max-off-peak autocorrelation ratio of the absolute encoding scheme seen in Figure 3(a). However, when employing the relative encoding- $\mathrm{B}$ and $\mathrm{C}$ schemes, the combined LA codes exhibited two pulses having a magnitude of 209 at the offsets of \pm 13 as well as the peak magnitude of 416 at the zero offset in their autocorrelation functions. Except for these two pulses at the offsets of \pm 13 , the autocorrelation values were similar to those generated with the aid of the relative encoding-A scheme of Figure 2(b).

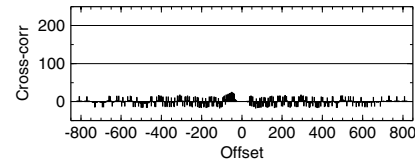

(a) Absolute encoding

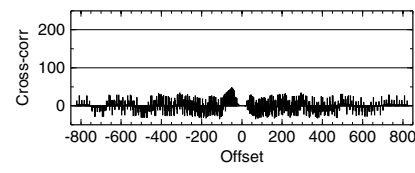

(c) Relative encoding - B

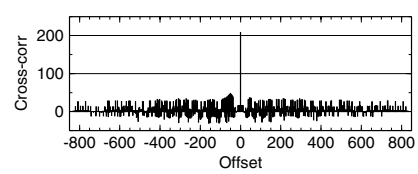

(b) Relative encoding - A

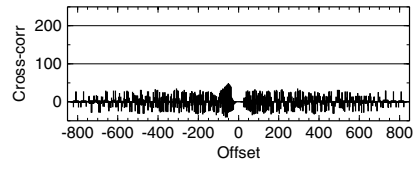

(d) Relative encoding - C
Figure 4: Crosscorrelations of $R_{\mathrm{w}_{2}, \mathrm{w}_{3}}$ for the combined LA $(847,38,16)$ codes using the 13-bit Barker sequence employing the four different encoding schemes illustrated in Figure 2.
The crosscorrelations of the combined LA $(847,38,16)$ code and the 13-bit Barker sequence employing the four different encoding schemes illustrated in Figure 2 are depicted in Figure 4. All the encoding schemes, except for the relative encodingA scheme, exhibited zero crosscorrelation values, when the code offset was less than 13 chip durations. The maximum crosscorrelation magnitude was 24 for the absolute encoding scheme and 48 for the relative encoding schemes, when ignoring the peak magnitudes in the range of 207 to 209 at the offset of zero or 13 recorded for the relative encoding scheme-A of Figure 2(b).

At this stage we may conclude that these combined LS codes using the 13-bit Barker sequence are capable of increasing their duty ratio considerably, although they do not exhibit a strict zero correlation zone. Nevertheless, the crosscorrelations of these combined codes employing the absolute encoding and the relative encoding $\mathrm{B} / \mathrm{C}$ schemes of Figure 2 exhibit a zero zone, when the relative code offset is less than the length of the Barker sequence involved. The ratio of their off-peak autocorrelation magnitude in comparison to the peak autocorrelation value is comparable to that of the Barker codes used. Hence, when we employ a better secondary spreading sequence, than the Barker sequences in terms of the associated aperiodic correlation properties, we may achieve a perfect IFW near zero offset. In the next section such a code family known as the set of LS codes is investigated and its combination with LA codes is studied, which are referred to as LAS codes.

\section{POLYPHASE LS CODES}

There exists a specific family of LS codes [6], which exhibits an IFW - a property similar to that of the family of LA codes. However, the strategy of LS codes adopted for achieving an IFW is quite different from that of LA codes. Specifically, LS codes exploit the properties of so-called orthogonal complementary sets $[6,11]$. To demonstrate further, let us introduce the notation of $\operatorname{LS}\left(N, P, W_{0}\right)$ for denoting the family of LS codes generated by applying a $P \times P$ dimensional Walsh-Hadamard matrix to an orthogonal complementary set of length $N$, while inserting $W_{0}$ number of zeros at the centre of the LS code, as described in [6]. Then, the total length of the $\operatorname{LS}\left(N, P, W_{0}\right)$ code is given by $2 N P+W_{0}$ and the number of available codes is given by $2 P$. It is known [12] that there exist binary complementary pairs of lengths $N=1,2$, $4,8,10,16,20,26,32,40,52,64,80,100$ for $N \leq 100$. On the other hand, there exist polyphase complementary pairs for all values of $N \leq 100$. Hence, LS codes based on polyphase complementary codes give us more flexibility in terms of the parameter $N$ determining the total code length of $2 N P+W_{0}$.

Since the construction method of binary LS codes is described in [6], let us concentrate our attention on the employment of polyphase orthogonal complementary sets $[12,13]$ for the generation of LS codes. The aperiodic correlation $R_{\mathrm{ab}}$ of two polyphase sequences $\mathbf{a}$ and $\mathbf{b}$ is defined as [13]:

$$
R_{\mathbf{a b}}[k] \triangleq \sum_{n=0}^{N+1-n} \mathbf{a}[n] \mathbf{b}^{*}[n+k],
$$

where $\mathbf{b}^{*}[i]$ is the complex conjugate of $\mathbf{b}[i]$. For a given 
polyphase complementary pair, $\left\{\mathbf{c}_{0}, \mathbf{s}_{0}\right\}$, of length $N$, one of the corresponding mate pairs can be written as:

$$
\begin{aligned}
& \mathbf{c}_{1}=\tilde{\mathbf{s}}_{0}^{*}, \\
& \mathbf{s}_{1}=-\tilde{\mathbf{c}}_{0}^{*},
\end{aligned}
$$

where $\tilde{\mathbf{s}}_{0}$ denotes the reversed sequence and $-\mathbf{s}_{0}$ the negated version of $\mathbf{s}_{0}$, respectively. Note that in (4) and (5) additional complex conjugation is required for polyphase complementary sequences in deriving the corresponding mate pair in comparison to binary complementary sequences [6]. Having obtained a polyphase complementary pair and its corresponding mate pair, we can employ the construction method of [6] for generating a family of LS codes. The LS codes generated exhibit an IFW, where we have $R_{\mathrm{ww}^{\prime}}[k]=0$ for $|k| \leq \min \left\{N-1, W_{0}\right\}$. Hence, we may maintain $W_{0}=N-1$ in order to reduce the total length of the LS codes, while providing as long an IFW as possible.

For example, the LS $(5,2,4)$ codes can be generated based on the quadriphase complementary pair of [12]:

$$
\begin{aligned}
& \mathbf{c}_{0}=+1+j-j-1+j \\
& \mathbf{s}_{0}=+1+1+1+j-j .
\end{aligned}
$$

Upon applying (4) and (5) to (6) and (7), the corresponding mate pair can be obtained as:

$$
\begin{aligned}
& \mathbf{c}_{1}=\tilde{\mathbf{s}}_{0}^{*}=+j-j+1+1+1 \\
& \mathbf{s}_{1}=-\tilde{\mathbf{c}}_{0}^{*}=+j+1-j+j-1 .
\end{aligned}
$$

Upon invoking the $2 \times 2$ Hadamard expansion of [6] in the context of the above quadriphase orthogonal complementary pairs, we can generate a family of four $\operatorname{LS}(5,2,4)$ codes, $\mathbf{w}_{p}$, where $p=0, \cdots, 3$. The corresponding aperiodic correlation values are depicted in Figure 5. All four different codes of

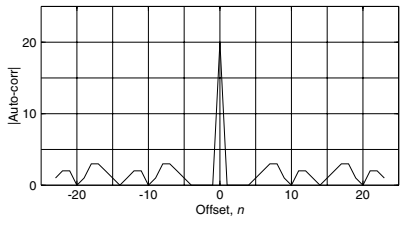

(a) Autocorrelation

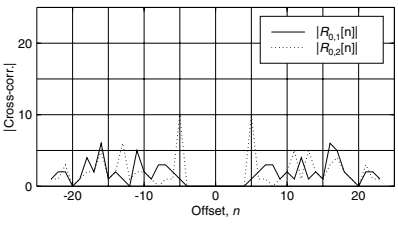

(b) Crosscorrelations
Figure 5: The correlation magnitudes of $\operatorname{LS}(5,2,4)$ codes. (a) All four codes exhibit the same autocorrelation magnitude. (b) The crosscorrelation magnitudes exhibit two different groups of behaviors.

the $\operatorname{LS}(5,2,4)$ family exhibited the same autocorrelation magnitudes, namely that seen in Figure 5(a). It can be observed in Figure 5 that the off-peak autocorrelation $R_{p}[n]$ becomes zero, when $|n| \leq W_{0}=4$. Even though it is not depicted in Figure 5(a), it was observed that $R_{0}[n]+R_{1}[n]=0$ and $R_{2}[n]+$ $R_{3}[n]=0$, implying that both $\left\{\mathbf{w}_{0}, \mathbf{w}_{1}\right\}$ and $\left\{\mathbf{w}_{2}, \mathbf{w}_{3}\right\}$ are polyphase complementary pairs. In fact, in general $2 P$ number of $\operatorname{LS}\left(N, P, W_{0}\right)$ codes constitute a polyphase complementary set [12]. This can be readily shown using the Hadamard expansion scheme of [6] employed for generating the LS codes. The crosscorrelation magnitudes $\left|R_{p, q}[n]\right|$ depicted in Figure 5(b) are also zero, when $|n| \leq W_{0}=4$. It was observed during our investigations that the crosscorrelations could be classified into two groups on the basis of their magnitudes, where $\left|R_{0,1}[n]\right|$ and $\left|R_{1,2}[n]\right|$ formed one group, while the other crosscorrelations, such as $\left|R_{0,2}\right|$, forming the other group. As seen in Figure 5(b), the latter group exhibited a relatively high correlation magnitude of $2 N(P-1)$ at the code offset of $n=N$. This is because when an LS code $\mathbf{w}_{p}$ is shifted by $N$ chips, $2(P-1)$ number of base complementary codes constituting the code $\mathbf{w}_{p}$ do overlap with those in a non-shifted code $\mathbf{w}_{q}$, where $P \leq q<2 P$, provided that $W_{0} \geq N$. When $W_{0}=N-1$, the magnitude of $2 N(P-1)$ is perturbed by an additive value of $\pm \mathbf{c}_{0}[N-1] \mathbf{s}_{0}^{*}[0]$, which happens to be zero for the $\operatorname{LS}(5,2,4)$ codes concerned. From the observations made in regards to the aperiodic correlations we may conclude that the quadriphase $\mathrm{LS}(5,2,4)$ codes exhibit an IFW within \pm 4 chip offsets.

In this section we demonstrated that $\operatorname{LS}\left(N, P, W_{0}\right)$ codes can be constructed for almost any code-length related parameter $N$ by employing polyphase sequences as well as binary sequences. Since LS codes exhibit an IFW, they constitute a good choice for amalgamation with the LA codes of Section 2 for increasing the duty ratio and hence the size of the code family. These combined codes constitute the topic of the next section.

\section{DESIGN CONSIDERATIONS ON LAS CODES}

We observed in Section 2 that the main problems associated with applying LA codes in practical CDMA system are their low duty ratio and the resultant small number of available codes. A specific family of LAS codes [6] solves this problem by combining the LA codes of Section 2 and the LS codes of Section 3 , in an effort to generate a large number of spreading codes having a high duty ratio, while maintaining attractive correlation properties.

Let us denote by $\operatorname{LAS}\left(L, M, K ; N, P, W_{0}\right)$ the combined code generated from the $\operatorname{LA}(L, M, K)$ and $\operatorname{LS}\left(N, P, W_{0}\right)$ codes by employing the absolute encoding method of Figure 2(a). For the sake of preserving the original IFW size of the constituent $\operatorname{LS}\left(N, P, W_{0}\right)$ code when combined with an $\operatorname{LA}(L, M, K)$ code employing the absolute encoding scheme, the length of the LS code - including $W_{0}$ number of trailing zeros - should not exceed the minimum pulse spacing $M$ of the LA code, requiring that:

$$
2 P N+2 W_{0} \leq M
$$

This is because if we have $2 P N+2 W_{0}>M$, the LS sequence associated with the adjacent pulse of the LA code interferes with the LS sequence related to the current pulse even when the code offset is less than $W_{0}$. The family size $K^{\prime}$ of the combined $\operatorname{LAS}\left(L, M, K ; N, P, W_{0}\right)$ codes is given by $K^{\prime}=$ $2 P K$. The $i$-th LAS code, where we have $0 \leq i<2 P K$, can be generated by combining the $k=\lfloor i /(2 P)\rfloor$-th LA code and the $(i-2 P k)$-th LS code.

Since there are $2 N P$ number of pulses having the magnitude of unity at each of the $K$ number of LA pulses in the $\operatorname{LAS}\left(L, M, K ; N, P, W_{0}\right)$ codes, the duty ratio $D$ of the LAS 
code is given by:

$$
D=\frac{2 N P K}{L} .
$$

For the sake of achieving the highest possible duty ratio, while maintaining an IFW of $\pm d$ chip durations, let us set $W_{0}=$ $N-1=d$ and choose an $M$ value obeying $M \geq M_{0}=$ $2 P N+2 W_{0}=2(P N+N-1)$. Since the code length $L$ of an LA code does not increases monotonically as the pulse spacing $M$ increases, as seen in Figure 1, sometimes it is better to choose $M>M_{0}$. The required IFW size, and hence the value of $d$, is determined by $d \geq \tau_{\max } f_{c}$, where $\tau_{\max }$ is the maximum channel-induced propagation delay and $f_{c}=T_{c}^{-1}$ is the chip rate. Once the value of $d$ is fixed, we have two independent design parameters, namely $P$ and $K$. Since the desired data rate is usually one of the most salient design constraints of the communication systems, there is typically a constraint on the total length $L$ of the LAS codes used depending on the modulation constellation size and the number of subcarriers employed in multi-carrier LAS-CDMA systems [4]. Table 1 depicts several LAS code designs contrived for the fixed IFW size of $d=4$ and $K=16$ LA pulses.

It can be observed in Table 1 that even though the minimum value of $M$ is given by $M_{0}=2\left(P N+W_{0}\right)=18$ for $P=1$, a value of $M=19$ was used in the design, since it resulted in a shorter LAS code length of 424 in comparison to 461 found for $M=18$. The duty ratio of the LAS codes in Table 1 increases, as the value of $P$ increases. Our modified pulse positioning scheme introduced in Section 2 produces consistently higher duty ratios, than those generated by employing the original scheme of [4]. However, the relative advantage of our scheme expressed in terms of the achievable duty ratios decreases, as $P$ increases. It should be noted that the reduction of the LAS code length not only increases the achievable duty ratio, but also improves the signaling efficiency by reducing the modulation symbol period $T_{s}$, which can be expressed as $T_{s}=L T_{c}$, where $T_{c}$ is the chip duration. For example, it can be shown that the LAS-CDMA modem employing our modified pulse positioning scheme is capable of transmitting $41 \%$ more symbols in comparison to that by employing the original pulse positioning scheme of [4], when $P=1$.

The length $N$ of the binary complementary pairs satisfying the condition $N \geq W_{0}+1=5$ is $N=8$. One can employ the binary $\operatorname{LS}(8, P, 4)$ codes, rather than the quadriphase $\operatorname{LS}(5, P, 4)$ codes of Table 1, which results in considerably longer LAS codes, than those generated using our design. For example, the binary LAS $(748,40,16 ; 8,2,4)$ codes are $33.8 \%$ longer, than the quadriphase $\operatorname{LAS}(559,28,16 ; 5,2,4)$ codes.

\section{CONCLUSIONS}

Having investigated the construction of LA codes, we proposed a modified pulse positioning scheme allowing both odd pulse spacing as well as even pulse spacing, in an effort to reduce the LA code length and to increase the achievable duty ratio. We also observed that the number $K$ of LA pulses does not have to obey the relationship of $K=2^{n}$, a code length satisfying $K=4 n$ for any positive integer $n$ is also acceptable. A total of three relative encoding schemes were involved

\begin{tabular}{rrr|rrrrrr}
\hline \hline$N$ & $W_{0}$ & \multicolumn{1}{c|}{$P$} & \multicolumn{1}{c}{$M$} & \multicolumn{1}{c}{$K^{\prime}$} & \multicolumn{1}{c}{$L$} & \multicolumn{1}{c}{$L^{\prime}$} & \multicolumn{1}{c}{$D$} & $D^{\prime}$ \\
\hline 5 & 4 & 1 & 19 & 32 & 424 & 713 & 37.7 & 22.4 \\
5 & 4 & 2 & 28 & 64 & 559 & 761 & 57.2 & 42.0 \\
5 & 4 & 4 & 48 & 128 & 875 & 995 & 73.1 & 64.3 \\
5 & 4 & 8 & 88 & 256 & 1514 & 1619 & 84.5 & 79.1 \\
5 & 4 & 16 & 168 & 512 & 2794 & 2899 & 91.6 & 88.3 \\
5 & 4 & 32 & 328 & 1024 & 5354 & 5459 & 95.6 & 93.8 \\
\hline \hline
\end{tabular}

Table 1: LAS code design examples. The size of the IFW is \pm 4 chips and the number of LA pulses is $K=16 . K^{\prime}$ represents the number of LAS codes available. $L$ and $L^{\prime}$ are the length of LAS codes employing the improved pulse positioning algorithm of Section 2-A and that of the original algorithm, respectively. Finally, $D$ and $D^{\prime}$ are the corresponding duty ratios expressed in percentage.

for code combining, where an LA code was amagamated with a short secondary spreading code. In the context of LS code design we investigated the use of polyphase complementary pairs and found that LS codes can be constructed for almost any base length value $N$. A set of LAS code design examples demonstrated that the added design flexibility owing to the proposed schemes may result in a considerable capacity increase as well as an increased duty ratio for the family of LAS codes. Our future research will study MC-CDMA schemes using LAS codes.

\section{REFERENCES}

[1] R. L. Peterson, R. E. Ziemer, and D. E. Borth, Introduction to Spread Spectrum Communications. Prentice Hall International Editions, 1995.

[2] M. K. Simon, J. K. Omura, R. A. Scholtz, and B. K. Levitt, Spread Spectrum Communications Handbook. New York, USA: McGraw-Hill, 1994.

[3] P. Fan and L. Hao, "Generalised orthogonal sequences and their applications in synchronous cdma systems," IEICE Trans. Fundamentals, vol. E83-A, pp. 2054-2069, Nov. 2000.

[4] D. Li, "A high spectrum efficient multiple access code," Chinese Journal of Electronics, vol. 8, pp. 221-226, Jul. 1999.

[5] D. Li, "Scheme for spread spectrum multiple access coding." US Patent, US 6,331,997 B1, Dec. 2001.

[6] S. Stańczak, H. Boche, and M. Haardt, "Are LAS-codes a miracle?," in GLOBECOM '01, vol. 1, (San Antonio, Texas), pp. 589-593, IEEE, Nov. 2001.

[7] S. Stańczak and H. Boche, "Sequences with small aperiodic correlations in the vicinity of the zero shift," in 4th International ITG Conference on Source and Channel Coding, (Berlin), pp. 183-190, ITG, VDE, Jan. 2002.

[8] J. J. van Lint and R. M. Wilson, A Course in Combinatorics. New York: Cambridge University Press, 1993.

[9] T. Beth, D. Jungnickel, and J. Lenz, Design Theory, vol. 1. Cambridge University Press, 2nd ed., 1999.

[10] R. H. Barker, "Group synchronizing of binary digital systems," in Communication Theory, pp. 273-287, 1953.

[11] C.-C. Tseng and C. L. Liu, "Complementary sets of sequences," IEEE Transactions on Information Theory, vol. 18, pp. 644-652, Sep. 1972.

[12] R. L. Frank, "Polyphase complementary codes," IEEE Transactions on Information Theory, vol. 26, pp. 641-647, Nov. 1980.

[13] R. Sivaswamy, "Multiphase complementarty codes," IEEE Transactions on Information Theory, vol. 24, pp. 546-552, Sep. 1978. 